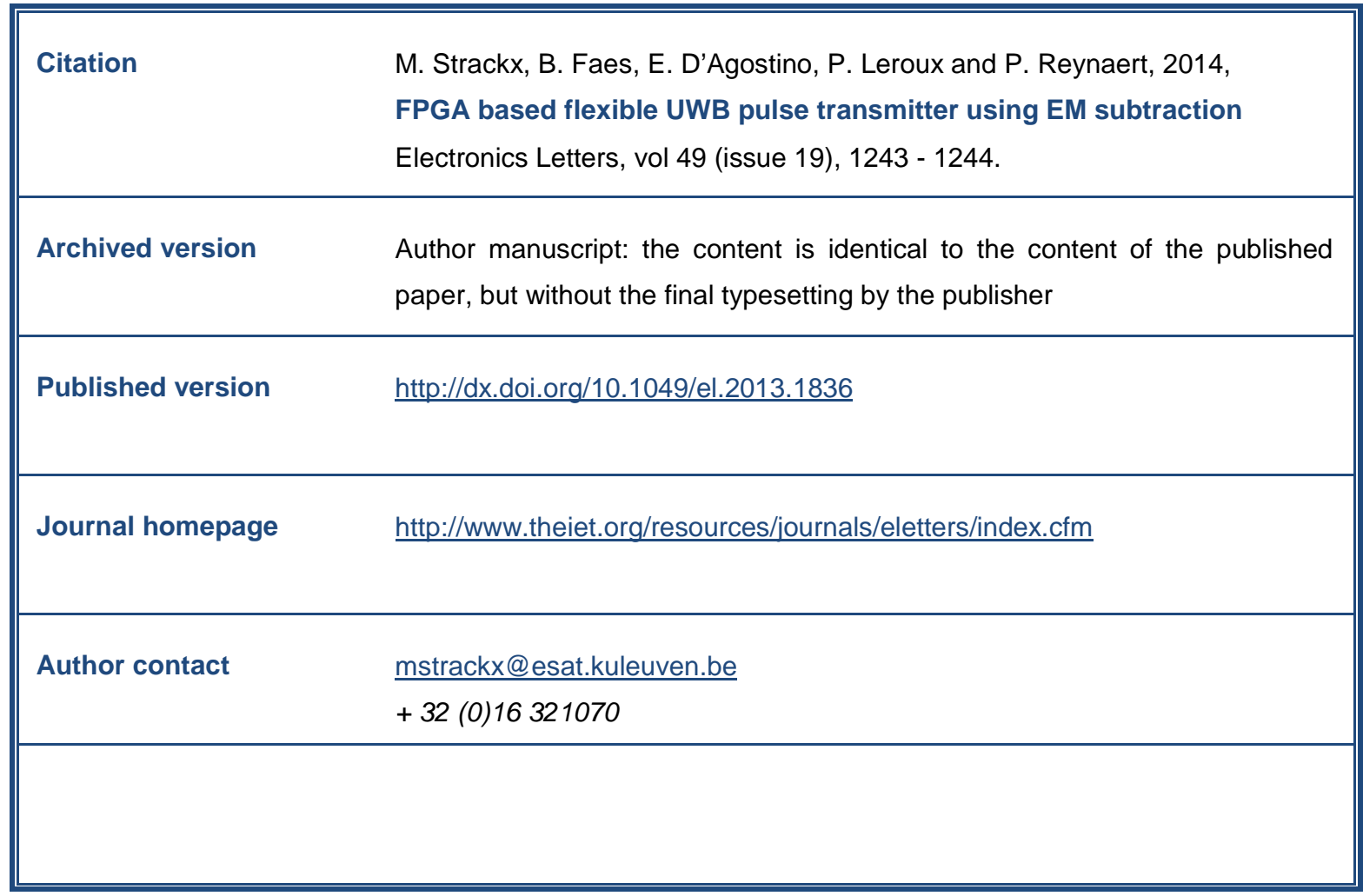

(article begins on next page) 


\section{FPGA based flexible UWB pulse transmitter using EM subtraction}

\section{Strackx, B. Faes, E. D’Agostino, P. Leroux and P. Reynaert}

An all-digital FPGA based electromagnetic (EM) subtraction technique for direct UWB pulse generation is presented. Using this technique it is possible to generate gaussian pulses, monocycles or doublets with high flexibility using a single printed circuit board (PCB) layout. User controlled parameters include: amplitude, pulse-width, pulse-type, repetition rate or even modulation. Measurements indicate a gaussian pulse with $1.24 \mathrm{~V}$ peak amplitude and a $10 \%$ pulsewidth of $670 \mathrm{ps}$ can be transmitted at a repetition frequency of $100 \mathrm{MHz}$. The corresponding $-10 \mathrm{~dB}$ bandwidth is $2.8 \mathrm{GHz}$

Introduction: Over the last decade, Ultra-WideBand (UWB) radio is commonly proposed for short-range high data rate communications [1] or for radar applications in the industrial [2] and medical fields. However, widespread implementation is still ongoing. This work contributes in providing an easy implementable development platform for UWB signal generation with higher flexibility compared to other transmitters. The key innovation of the work is the usage of a field programmable gate array (FPGA) together with an electromagnetic (EM) subtraction technique to form different gaussian pulse shapes in a direct way. Two general transmitter implementations exist to date. CMOS integrated circuits can be considered for full-custom designs while on the other hand transmitters can be composed using commercial off-the-shelf (COTS) components. The latter are implemented with step recovery diodes (SRD) [2] or mesfets to generate steep edges and provide flexibility to some extent. An all-digital FPGA based implementation combines the high flexibility of CMOS designs together with rapid and easy implementation.

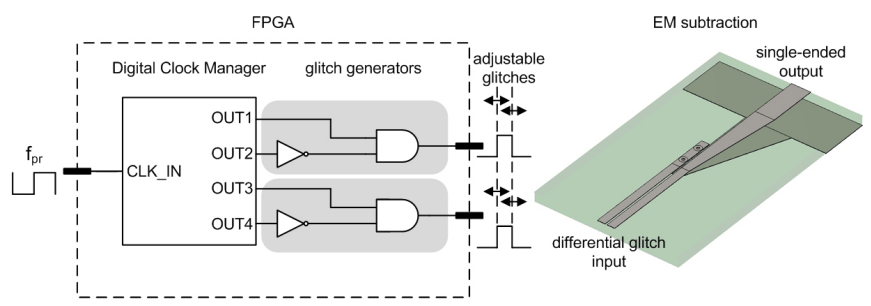

Fig. 1 Block diagram of the FPGA based UWB pulse transmitter with EM subtraction on PCB level using a CPS to MS transition

Architecture: The architecture of the pulse generator is presented in Fig. 1. An external clock signal controls the pulse repetition frequency of the transmitted UWB pulses. For optional increased flexibility it is also possible to use a frequency synthesiser of the FPGA to change this parameter. The clock signal is used internally to drive a digital clock manager (DCM) which includes a phase locked loop (PLL) to lower the jitter at the output and to generate several out of phase components. The phase shifts of each DCM output can be controlled on-the-fly. In this way, the DCM can be considered as a digital to time converter (DTC) which benefits from CMOS scaling. When using a more recent technology node, timing resolution will increase. Also depending on the application, it is possible to choose a different FPGA with higher or lower timing resolution. Each pair of DCM outputs is combined in a glitch generator which has both reconfigurable rising and falling edges. Each glitch can now be shifted over time as well as having an adjustable pulse-width

EM subtraction: Both glitches are transmitted by a differential coplanar stripline (CPS) which supresses the common mode component and passes through the differential one. Mathematically this is the equivalent of a subtraction. One of the striplines is connected to the bottom side of the PCB and forms the new ground plane of the microstrip (MS) transmission line. Both groundplane and other stripline are linearly tapered to minimise insertion and reflection losses. A general mathematical understanding of this type of transition is given in [3]. Compared to [3], the transition is altered for the UWB application using the following adjustments: linear tapering profiles, less vias and tapering of the top stripline. Full-wave EM
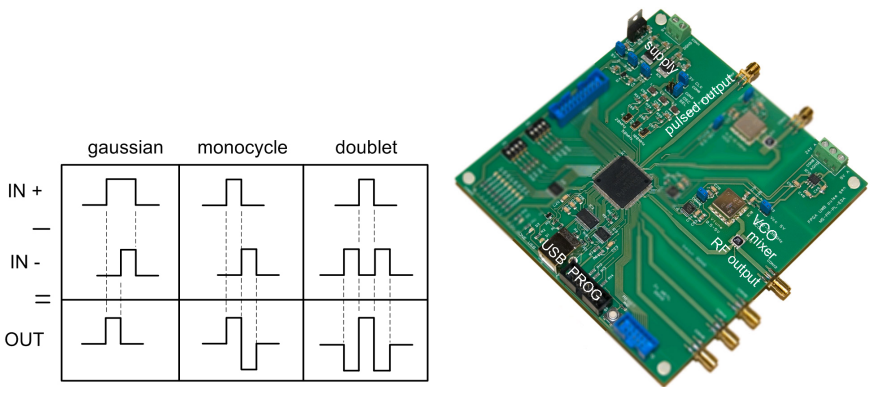

Fig. 2 EM subtraction technique for gaussian pulse generation and PCB of the FPGA based UWB transmitter

simulations indicate a return loss better than $10 \mathrm{~dB}$ in the $1.5-10 \mathrm{GHz}$ band with an insertion loss less than $2 \mathrm{~dB}$ on a FR4 substrate. Using the proposed EM subtraction technique, the pulse generator is capable of generating gaussian pulses, monocycles and doublets with the same architecture. The different pulses are generated based on user adjustable time delays. Fig. 2 shows the operating principle of the proposed EM subtraction technique to form different kinds of pulse shapes. The amplitude of the generated pulses can also be controlled by adjusting the slew-rate setting of the FPGA. For doublet generation, an additional pair of DCM outputs is added in combination with a glitch generator. Two glitch generator outputs then need to be combined using an OR gate for $I N-$. For better FCC compliant pulse generation, a voltage controlled oscillator (VCO) was added in a second test structure with a mixer for frequency translation. The oscillation frequency can also be controlled from the FPGA, providing additional flexibility. The maximum centre frequency for up-conversion is $5.5 \mathrm{GHz}$ in this design.

Measurement results: The FPGA output is directly connected via the CPS-MS transition to a high-frequency oscilloscope. The measured UWB pulses are shown in Fig. 3 both in time and frequency domains. These are generated using the smallest time offset and highest slew rate setting on a Xilinx Spartan 6 FPGA to show the performance. Wider pulses with narrower frequency content can easely be generated with larger time offsets. The measured gaussian pulse has a $10 \%$ pulse-width of 670 ps with a peak amplitude of $1.24 \mathrm{~V}$, achieving a $10 \mathrm{~dB}$ bandwidth of $2.8 \mathrm{GHz}$. The gaussian monocycle has a $10 \%$ pulse-width of 1.48 ns with a peakto-peak amplitude of $2.64 \mathrm{~V}$, achieving a $10 \mathrm{~dB}$ bandwidth of $2.0 \mathrm{GHz}$. The gaussian doublet has a $10 \%$ pulse-width of $1.67 \mathrm{~ns}$ with a peak-topeak amplitude of $1.74 \mathrm{~V}$, achieving also a $10 \mathrm{~dB}$ bandwidth of $2.0 \mathrm{GHz}$. These signals indicate the high flexibility of the transmitter. However, the generated pulses still have some low frequency components which are difficult to transmit with small UWB antennas. For this reason, an additional up-conversion can be performed to $5.5 \mathrm{GHz}$ as an example. The example up-converted pulse is shown in Fig. 4, both in time and frequency domains. The pulse has a $10 \%$ width of $2.02 \mathrm{~ns}$ with a peak-topeak amplitude of $482 \mathrm{mV}$, achieving a $10 \mathrm{~dB}$ bandwidth of $2.3 \mathrm{GHz}$. The signal energy present in the $1-1.6 \mathrm{GHz}$ band can be further reduced using a proper UWB antenna or filter. Table 1 lists all the measured parameters and compares performance with $65 \mathrm{~nm}$ CMOS [1], SRD on PCB [2], $0.13 \mu \mathrm{m}$ CMOS [4] and $0.18 \mu \mathrm{m}$ CMOS [5, 6] designs. Compared to CMOS designs, similar performance is achieved. Compared to PCB designs with discrete components, higher flexibility is demonstrated.
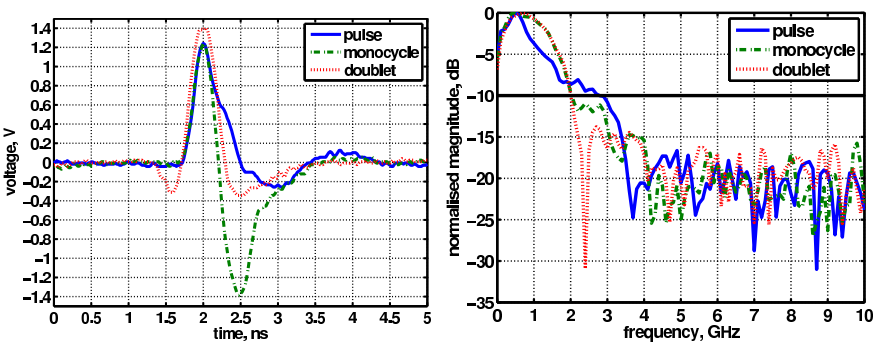

Fig. 3 Measured gaussian pulse, monocycle and doublet in time and frequency domains 

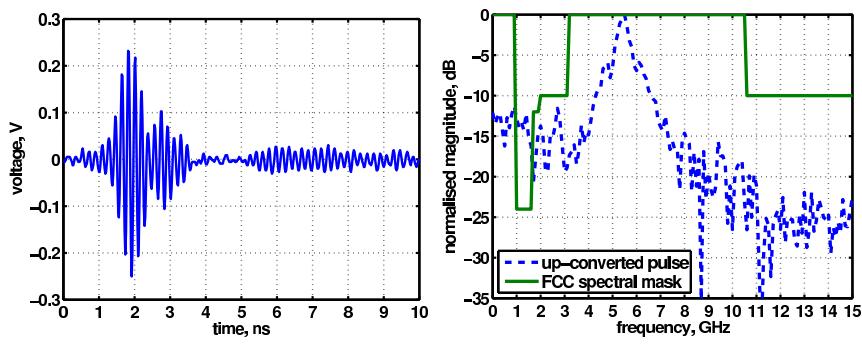

Fig. 4 Measured up-converted pulse in time and frequency domains for transmission with UWB antennas

Table 1: Performance comparison to prior work

\begin{tabular}{|l|l|l|l|l|l|}
\hline ref & $T_{10 \%}$ & $V_{p t p}$ & $f_{-10 d B}$ & $f_{c}$ & Type \\
\hline$[1]$ & $2.5 \mathrm{~ns}$ & $91 \mathrm{mV}$ & $1.4 \mathrm{GHz}$ & $5 \mathrm{GHz}$ & up-converted \\
\hline$[2]$ & $450 \mathrm{ps}$ & $5.8 \mathrm{~V}$ & $3.7 \mathrm{GHz}$ & - & monocycle \\
\hline$[4]$ & $1.0 \mathrm{~ns}$ & $240 \mathrm{mV}$ & $2 \mathrm{GHz}$ & $4 \mathrm{GHz}$ & higher order \\
\hline$[5]$ & $500 \mathrm{ps}$ & $673 \mathrm{mV}$ & $4.5 \mathrm{GHz}$ & $8.2 \mathrm{GHz}$ & up-converted \\
\hline$[6]$ & $1.75 \mathrm{~ns}$ & $1.25 \mathrm{~V}$ & $1.4 \mathrm{GHz}$ & $4.1 \mathrm{GHz}$ & higher order \\
\hline \hline This work & $670 \mathrm{ps}$ & $1.24 \mathrm{~V}$ & $2.8 \mathrm{GHz}$ & $0.5 \mathrm{GHz}$ & gaussian pulse \\
\hline This work & $1.48 \mathrm{~ns}$ & $2.64 \mathrm{~V}$ & $2.0 \mathrm{GHz}$ & $0.5 \mathrm{GHz}$ & monocycle \\
\hline This work & $1.67 \mathrm{~ns}$ & $1.74 \mathrm{~V}$ & $2.0 \mathrm{GHz}$ & $0.7 \mathrm{GHz}$ & doublet \\
\hline This work & $2.02 \mathrm{~ns}$ & $482 \mathrm{mV}$ & $2.3 \mathrm{GHz}$ & $5.5 \mathrm{GHz}$ & up-converted \\
\hline
\end{tabular}

Conclusion: This letter presents an FPGA based flexible UWB pulse transmitter. Using the proposed EM substraction technique, gaussian pulses, monocycles and doublets can be generated by a single transmission line structure on the PCB. Due to the high flexibility, this technique offers a rapid prototyping alternative compared to CMOS designs. As a proof-ofconcept, an up-conversion is also performed such that generated pulses can be transmitted with antennas and comply with spectral emission limits.
Acknowledgment: This research is supported by the Belgian Nuclear Research Centre SCK•CEN.

One or more of the figures in this Letter are available in colour online.

M. Strackx, P. Leroux and P. Reynaert (KU Leuven, ESAT-MICAS, Leuven, Belgium)

E-mail: maarten.strackx@esat.kuleuven.be

E. D’Agostino $(S C K \bullet C E N, E H S-R D C, M o l$, Belgium $)$

B. Faes and P. Leroux (KU Leuven, ESAT-EEET, MOBILAB-RELIC, Geel, Belgium)

\section{References}

1 Youngmin, P. and Wentzloff, D.D.: 'An All-Digital 12 pJ/Pulse IR-UWB Transmitter Synthesized From a Standard Cell Library', IEEE Journal of Solid-State Circuits, vol. 46, no. 5, pp. 1147-1157, May 2011

2 Han, J. and Nguyen C.: 'On the development of a compact sub-nanosecond tunable monocycle pulse transmitter for UWB applications', IEEE Tran. on Microwave Theory and Techniques, vol. 54, no. 1, pp. 285-293, Jan. 2006

3 Young-Gon, K., Dong-Sik, W., Kang Wook, K. and Young-Ki, C.: 'A New Ultra-wideband Microstrip-to-CPS Transition', IEEE/MTT-S Int. Microwave Symposium, pp. 1563-1566, Jun. 2007

4 Xia, L., Huang, Y. and Hong, Z.: 'Low power amplitude and spectrum tunable IR-UWB transmitter', Electronics Letters, vol. 44, no. 20, pp. 12001201, Sep. 2008

5 Sanghoon, S., Dong-Wook, K. and Songcheol, H.: 'A CMOS UWB Pulse Generator for 6-10 GHz Applications', IEEE Microwave and Wireless Components Letters, vol. 19, no. 2, pp. 83-85, Feb. 2009

6 Norimatsu, T., Fujiwara, R., Kokubo, M., Miyazaki, M., Maeki, A. and Ogata, Y.: 'Kobayashi, S.; Koshizuka, N.; Sakamura, K., "A UWB-IR Transmitter With Digitally Controlled Pulse Generator', IEEE Journal of Solid-State Circuits, vol. 42, no. 6, pp. 1300-1309, Jun. 2007 